\title{
Automorphisms of non-spherical buildings have unbounded displacement
}

\author{
Peter Abramenko Kenneth S. Brown
}

\begin{abstract}
If $\phi$ is a nontrivial automorphism of a thick building $\Delta$ of purely infinite type, we prove that there is no bound on the distance that $\phi$ moves a chamber. This has the following group-theoretic consequence: If $G$ is a group of automorphisms of $\Delta$ with bounded quotient, then the center of $G$ is trivial.
\end{abstract}

Keywords : building, automorphism, displacement, center

MSC 2000: 51E24, 20E42

\section{Introduction}

A well-known folklore result says that a nontrivial automorphism $\phi$ of a thick Euclidean building $X$ has unbounded displacement. Here we are thinking of $X$ as a metric space, and the assertion is that there is no bound on the distance that $\phi$ moves a point. [For the proof, consider the action of $\phi$ on the boundary $X_{\infty}$ at infinity. If $\phi$ had bounded displacement, then $\phi$ would act as the identity on $X_{\infty}$, and one would easily conclude that $\phi=$ id.] In this note we generalize this result to buildings that are not necessarily Euclidean. We work with buildings $\Delta$ as combinatorial objects, whose set $\mathcal{C}$ of chambers has a discrete metric ("gallery distance"). We say that $\Delta$ is of purely infinite type if every irreducible factor of its Weyl group is infinite.

Theorem. Let $\phi$ be a nontrivial automorphism of a thick building $\Delta$ of purely infinite type. Then $\phi$, viewed as an isometry of the set $\mathcal{C}$ of chambers, has unbounded displacement.

It is possible to prove the theorem by using the Davis realization of $\Delta$ as a CAT(0) metric space [3] and arguing as in the Euclidean case. (But more 


The next result allows one to talk about separation of cells by walls. It is part of Theorem 2.80 in [1, Section 2.6], and it can also be deduced from Proposition 5 in [2, Section V.4.6].

Lemma 1.3. If $H$ is a wall and $A$ is a cell, then either $A$ is contained in $H$ or $A$ is contained in one of the two open halfspaces determined by $H$.

We turn now to reflections. The following lemma is an easy consequence of the stabilizer calculation in [1, Theorem 2.80] or [2, Section V.4.6].

full screen

close

Lemma 1.4. For each wall $H \in \mathcal{H}$, there is a unique nontrivial element $s_{H} \in W$ that fixes $H$ pointwise.

We call $s_{H}$ the reflection with respect to $H$. In view of a fact stated above, we have $s_{H_{s}}=s$ for all $s \in S$. Thus $S$ is the set of reflections with respect to the walls in $\mathcal{H}_{0}$. It follows immediately from Lemma 1.4 that

$$
s_{w H}=w s_{H} w^{-1}
$$

for all $H \in \mathcal{H}$ and $w \in W$. Hence $w S w^{-1}$ is the set of reflections with respect to the walls of $w C_{0}$.

Corollary 1.5. For $s \in S$ and $w \in W, H_{s}$ is a wall of $w C_{0}$ if and only if $w^{-1} s w$ is in $S$.

Proof. $H_{s}$ is a wall of $w C_{0}$ if and only if $s$ is the reflection with respect to a wall of $w C_{0}$. In view of the observations above, this is equivalent to saying $s \in w S w^{-1}$, i.e., $w^{-1} s w \in S$.

Finally, we record some special features of the infinite case.

Lemma 1.6. Assume that $(W, S)$ is irreducible and $W$ is infinite.

(1) If two chambers $C, D$ have the same walls, then $C=D$.

(2) The Tits cone $X$ does not contain any pair $\pm x$ of opposite nonzero vectors.

Proof. (1) We may assume that $C=C_{0}$ and $D=w C_{0}$ for some $w \in W$. Then Corollary 1.5 implies that $C$ and $D$ have the same walls if and only if $w$ normalizes $S$. So the content of (1) is that the normalizer of $S$ in $W$ is trivial. This is a well known fact. See [2, Section V.4, Exercise 3], [4, Proposition 4.1], or [1, Section 2.5.6]. Alternatively, there is a direct geometric proof of (1); see [1, Exercises 3.121 and 3.122].

(2) This is a result of Vinberg [15, p. 1112, Lemma 15]. See also [1, Section 2.6.3] and [6, Theorem 2.1.6] for alternate proofs. 


\section{A lemma about Coxeter groups}

We begin with a geometric version of our lemma, and then we translate it into algebraic language.

Lemma 2.1. Let $(W, S)$ be an infinite irreducible Coxeter system with $S$ finite. If $C$ and $D$ are distinct chambers in the Tits cone, then $C$ has a wall $H$ with the following two properties:

(a) $H$ is not a wall of $D$.

(b) $H$ does not separate $C$ from $D$.

Proof. For convenience (and without loss of generality), we assume that $C$ is the fundamental chamber $C_{0}$. Define $J \subseteq S$ by

$$
J:=\left\{s \in S \mid H_{s} \text { is a wall of } D\right\},
$$

and set $L:=\bigcap_{s \in J} H_{s}$. Thus $L$ is the support of the face $A=A_{J}$ of $C$. By Lemma 1.6(1), $J \neq S$, hence $L \neq\{0\}$. Since $L$ is an intersection of walls of $D$, it is also the support of a face $B$ of $D$. Note that $A$ and $B$ are contained in precisely the same walls, since they have the same span $L$. In particular, $B$ is not contained in any of the walls $H_{s}$ with $s \in S \backslash J$, so, by Lemma $1.3, B$ is contained in either $U_{+}(s)$ or $U_{-}(s)$ for each such $s$.

Suppose that $B \subseteq U_{-}(s)$ for each $s \in S \backslash J$. Then, in view of the definition of $A=A_{J}$ by linear equalities and inequalities, $B \subseteq-A$. But $B$ contains a nonzero vector $x$ (since $B$ spans $L$ ), so we have contradicted Lemma 1.6(2). Thus there must exist $s \in S \backslash J$ with $B \subseteq U_{+}(s)$. This implies that $D \subseteq U_{+}(s)$, and the wall $H=H_{s}$ then has the desired properties (a) and (b).

We now prove the algebraic version of the lemma, for which we relax the hypotheses slightly. We do not even have to assume that $S$ is finite. Recall that $(W, S)$ is said to be purely infinite if each of its irreducible factors is infinite.

Lemma 2.2. Let $(W, S)$ be a purely infinite Coxeter system. If $w \neq 1$ in $W$, then there exists $s \in S$ such that:

(a) $w^{-1} s w \notin S$.

(b) $l(s w)>l(w)$.

Proof. Let $\left(W_{i}, S_{i}\right)$ be the irreducible factors of $(W, S)$, which are all infinite. Suppose the lemma is true for each factor $\left(W_{i}, S_{i}\right)$, and consider any $w \neq 1$ in $W$. Then $w$ has components $w_{i} \in W_{i}$, at least one of which (say $w_{1}$ ) is 

Proof. The second assertion follows at once from the first. To prove the first, set $\sigma(s):=w^{-1} s w \in K$ for $s \in J$, so that

$$
s w=w \sigma(s)
$$

for all $s \in J$. This equation and the definition of $J$ imply that $w$ is right $K$-reduced and hence that

$$
l(w v)=l(w)+l(v)
$$

for all $v \in W_{K}$. In particular, the (unique) longest element of the coset $w W_{K}$ is $u:=w w_{0}(K)$. To show that $u=w_{0}$, we need to show that $l(s u)<l(u)$ for all $s \in S$. If $s \in J$, we have $s u=w \sigma(s) w_{0}(K) \in w W_{K}$, so $l(s u)<l(u)$ because $u$ is the longest element of $w W_{K}$. And if $s \notin J$, then

$$
l(s u)=l\left(s w w_{0}(K)\right)<l(w)+l\left(w_{0}(K)\right)=l(u)
$$

because $l(s w)<l(w)$.

\section{Proof of the theorem}

In this section we assume familiarity with basic concepts from the theory of buildings $[1,8,9,13,16]$.

Let $\Delta$ be a building with Weyl group $(W, S)$, let $\mathcal{C}$ be the set of chambers of $\Delta$, and let $\delta: \mathcal{C} \times \mathcal{C} \rightarrow W$ be the Weyl distance function. (See [1, Section 4.8 or 5.1] for the definition and standard properties of $\delta$.) Recall that $\mathcal{C}$ has a natural gallery metric $d(-,-)$ and that

$$
d(C, D)=l(\delta(C, D))
$$

for $C, D \in \mathcal{C}$. Let $\phi: \Delta \rightarrow \Delta$ be an automorphism of $\Delta$ that is not necessarily type-preserving. Recall that $\phi$ induces an automorphism $\sigma$ of $(W, S)$. From the simplicial point of view, we can think of $\sigma$ (restricted to $S$ ) as describing the effect of $\phi$ on types of vertices. From the point of view of Weyl distance, $\sigma$ is characterized by the equation

$$
\delta(\phi(C), \phi(D))=\sigma(\delta(C, D))
$$

for $C, D \in \mathcal{C}$.

Our main theorem will be obtained from the following technical lemma:

Lemma 3.1. Assume that $\Delta$ is thick. Fix a chamber $C \in \mathcal{C}$, and set $w:=$ $\delta(C, \phi(C))$. Suppose there exists $s \in S$ such that $l(s w)>l(w)$ and $w^{-1} s w \neq \sigma(s)$. Then there is a chamber $D$ s-adjacent to $C$ such that $d(D, \phi(D))>d(C, \phi(C))$. 





\begin{abstract}
1
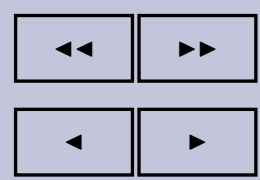

page $12 / 13$

go back

full screen

close

quit

Remark 4.5. For even $m=2 n$, type-preserving automorphisms $\phi$ of generalized $m$-gons with $\operatorname{disp} \phi=m-1$ arise as follows. Assume that there exists a vertex $x$ in the generalized $m$-gon $\Delta$ such that the ball $B(x, n)$ is fixed pointwise by $\phi$. Here $B(x, n)$ is the set of vertices with $d(x, y) \leq n$, where $d(-,-)$ now denotes the usual graph metric, obtained by minimizing lengths of paths. Recall that there are two types of vertices in $\Delta$ and that opposite vertices always have the same type since $m$ is even. Let $y$ be any vertex that does not have the same type as $x$. Then $y$ is at distance at most $n-1$ from some vertex in $B(x, n)$. Since $\phi$ fixes $B(x, n)$ pointwise, $d(y, \phi(y)) \leq 2 n-2$. So $C$ and $\phi(C)$ are not opposite for any chamber $C$ having $y$ as a vertex. Since this is true for any vertex $y$ that does not have the same type as $x, \operatorname{disp} \phi \neq m$ and hence, by Corollary 4.4(a), $\operatorname{disp} \phi=m-1$ if $\phi \neq \mathrm{id}$. Now it is a well-known fact (see for instance [14, Corollary 5.4.7]) that every Moufang $m$-gon possesses nontrivial type-preserving automorphisms $\phi$ fixing some ball $B(x, n)$ pointwise. (In the language of incidence geometry, these automorphisms are called central or axial collineations, depending on whether $x$ is a point or a line in the corresponding rank 2 geometry.) So for $m=4,6$, or 8 , all Moufang $m$-gons admit typepreserving automorphisms $\phi$ with $\operatorname{disp} \phi=m-1$.
\end{abstract}

\title{
References
}

[1] P. Abramenko and K. S. Brown, Buildings: Theory and Applications, Grad. Texts in Math. 248, Springer, New York, 2008.

[2] N. Bourbaki, Éléments de mathématique. Fasc. XXXIV. Groupes et algèbres de Lie. Chapitre IV: Groupes de Coxeter et systèmes de Tits. Chapitre V: Groupes engendrés par des réflexions. Chapitre VI: Systèmes de racines, Actualités Scientifiques et Industrielles, No. 1337, Hermann, Paris, 1968.

[3] M. W. Davis, Buildings are CAT(0), in Geometry and cohomology in group theory (Durham, 1994), London Math. Soc. Lecture Note Ser., vol. 252, Cambridge Univ. Press, Cambridge, 1998, pp. 108-123.

[4] V. V. Deodhar, On the root system of a Coxeter group, Comm. Algebra 10 (1982), no. 6, 611-630.

[5] J. E. Humphreys, Reflection Groups and Coxeter Groups, Cambridge Stud. Adv. Math., vol. 29, Cambridge University Press, Cambridge, 1990.

[6] D. Krammer, The Conjugacy Problem for Coxeter Groups, Groups Geom. Dyn. 3 (2009), 71-171. 
